\title{
REHIDRATACION POST-EJERCICIO CON DIFERENTES TIPOS DE BEBIDAS: AGUA PURA, BEBIDA DEPORTIVA Y AGUA DE JAMAICA
}

\author{
María de Lourdes Mayol Soto y Luis Fernando Aragón Vargas \\ Escuela de Educación Física y Deportes \\ Universidad de Costa Rica, San José, Costa Rica \\ E-mail: lulumayol@yahoo.com
}

\begin{abstract}
Resumen
Mayol Soto, .M. L. y Aragón Vargas, L. F. (2002). Rehidratación post-ejercicio con diferentes tipos de bebidas: agua pura, bebida deportiva y agua de Jamaica. Revista de Ciencias del Ejercicio y la Salud, 2(1), 41-54. El propósito del presente estudio fue determinar si el agua de jamaica (Hibiscus Sabdarifa L.), bebida muy consumida en México y considerada útil por su "propiedad diurética", es adecuada para la rehidratación después del ejercicio, comparándola con una bebida deportiva y el agua pura. En este estudio participaron 16 varones, entre los 17 y 23 años de edad, físicamente activos y estudiantes de la Universidad de Costa Rica. Los sujetos consumieron los tres tratamientos en tres días distintos, el mismo día de la semana. El orden de los tratamientos fue aleatorizado. Los participantes se presentaron a cada prueba en un estado adecuado de hidratación y después de un desayuno estandarizado y un breve descanso, realizaron un ejercicio intermitente en bicicleta dentro de un cuarto de clima controlado (30-35 ${ }^{\circ} \mathrm{C}$ y $85 \% \mathrm{HR}$ ) hasta alcanzar un $2.3 \%$ de pérdida de masa corporal. Después de descansar durante 30 min a temperatura ambiente consumieron el $150 \%$ de la pérdida de masa corporal de la bebida correspondiente a cada día, durante un período de $45 \mathrm{~min}$. Al término del período de rehidratación (0 min) se inició la recolección de muestras de orina y se continuó cada 30 min hasta completar 3 horas. Se determinó el volumen, la densidad y el color de cada una de las muestras. Los resultados de volumen se analizaron como el porcentaje del volumen ingerido. Para analizar los datos se utilizó estadística descriptiva y ANOVA de 2 vías de medidas repetidas en ambos factores. Aunque no hubo diferencias estadísticamente significativas en la producción total de orina ( $p=0.084)$, se encontró una interacción entre la producción de orina, la densidad y el color, con el tiempo $(p<0.001)$. Al analizar estas interacciones, se observó que aunque en un inicio se excretó en mayor proporción, la producción de orina con la bebida deportiva fue significativamente menor a los 150 y 180 min, además de que las orinas producidas por esta bebida fueron significativamente más densas y más oscuras a los 0, 150 y 180 min, indicando un mayor grado de conservación de líquido. A partir de los resultados obtenidos se puede concluir que el agua de jamaica no presentó un efecto diurético después de 3 horas de haber sido consumida por sujetos deshidratados por ejercicio. Aunque pareciera ser que la rehidratación es casi igual con las tres bebidas, la dinámica de producción y composición de la orina es diferente con cada una de las bebidas, además de que hacia el final del estudio se observó una tendencia de la bebida deportiva a conservarse más dentro del organismo. PALABRAS CLAVES: rehidratación postejercicio, diurético, diuresis, bebida deportiva, agua de jamaica, agua pura, producción de orina, conservación de líquido.
\end{abstract}

\section{INTRODUCCION}

En condiciones de reposo, el contenido de agua del cuerpo permanece relativamente estable y la pérdida de fluidos es equivalente al consumo de estos (Wilmore y Costill 1994). No obstante, cuando se realiza ejercicio, en condiciones ambientales extremas, las tasas de sudoración pueden exceder de 2 litros (Maughan, 1998) o hasta 3 litros por hora (Horswill, 1998).

Un consumo inadecuado de líquidos durante el ejercicio, afectará la regulación de la temperatura, la función cardiovascular y el 
metabolismo del músculo (Horswill, 1998). No obstante, el efecto negativo de la deshidratación en el rendimiento deportivo y en la salud, puede verse atenuado por el consumo de líquidos para compensar las pérdidas por sudoración (Maughan, 1998).

La cantidad de líquido que un individuo pierde puede ser estimada con un alto grado de confiabilidad si se miden los cambios en el peso corporal durante el período de tiempo en el que se da esta pérdida. Sin embargo, es más difícil estimar las pérdidas de electrolitos, ya que la composición del sudor es difícil de medir, además de que hay grandes variaciones entre individuos, y en el mismo individuo la composición del sudor varía en el tiempo. Los principales electrolitos que se pierden en el sudor son el sodio y el cloro, y el reemplazo de estos iones, sobre todo del sodio, es una prioridad. Sin embargo, debido a la gran variabilidad en la pérdida de electrolitos que ocurre en diferentes situaciones, se dificulta formular una recomendación general para compensar los electrolitos (Maughan, 1998).

Se han realizado varias investigaciones para determinar los requerimientos de agua y electrolitos para la recuperación después del ejercicio.

Nadel, Mack y Nose (1990) mostraron que el consumo de agua pura después del ejercicio, da como resultado una rápida caída de las concentraciones de sodio y la osmolaridad del plasma. Ambos factores son importantes en el balance de líquido, ya que reducen el estímulo de la sed y estimulan la producción de orina.

Maughan y Leiper (1995) demostraron que el grado de retención de líquido después del ejercicio aumenta cuando se consumen bebidas con una mayor concentración de sodio.

Posteriormente, Shirreffs, Taylor, Leiper y Maughan (1996) encontraron, que además de la cantidad de sodio, es importante tomar en cuenta el volumen consumido. Ellos demostraron que cuando se consume el $100 \%$ del volumen de líquido perdido durante el ejercicio, la excreción de orina puede producir un déficit cercano a los $0.5 \mathrm{~L}$ después de 1 hora de haber consumido el líquido. Consumos de 150\% y 200\% de la pérdida de líquidos, compensan las pérdidas por orina. Sin embargo, es necesario que el contenido de sodio de la bebida sea lo suficientemente alto (> $50 \mathrm{mmol} / \mathrm{l}$ ), para conservar el líquido durante más tiempo (Maughan, 1998).

Por lo tanto, el consumo de sodio después del ejercicio es indispensable; no obstante este sodio no necesariamente debe provenir de la bebida que se ingiera, sino que también puede obtenerse a partir de los alimentos. Así lo demostraron Maughan, Leiper y Shirreffs (1996), quienes observaron que la recuperación de líquidos después del ejercicio puede darse por la ingestión de agua pura si se consume un volumen suficiente junto con una comida que aporte cantidades significativas de electrolitos.

Otro aspecto que debe de tomarse en cuenta es el sabor de la bebida, ya que de esto dependerá el consumo voluntario de la misma. Es importante que el individuo consuma bebidas que sean de su agrado y sobre todo que estén disponibles. Por lo tanto, es de suma importancia determinar si las bebidas que más se consumen en determinada población son útiles para la rehidratación después del ejercicio. En un estudio realizado por Aragón-Vargas y Madriz-Dávila (2000), se determinó que el agua de coco, ampliamente consumida en Costa Rica, podría ser efectiva para la rehidratación después del ejercicio.

En un estudio realizado con agua de jamaica, la cual es ampliamente conocida y consumida en México por su "efecto diurético”, se observó que el grado de conservación de líquido es similar al de una bebida deportiva, por lo cual, muy lejos de presentar el efecto diurético, podría ser útil para la rehidratación después del ejercicio. En dicho estudio, se evaluó el grado de conservación del líquido producido por el agua de jamaica, comparándola con el agua 
pura y una bebida deportiva, en condiciones de reposo y bajo un nivel adecuado de hidratación. Se observó que el agua pura se excretó en mayor cantidad que las otras dos bebidas y existió una diferencia estadísticamente significativa a los $30 \mathrm{~min}$ y a las 2 horas de haber ingerido la bebida, momento en el cual ya se había excretado una cantidad equivalente al $109.64 \%$ del volumen de agua ingerido. Con las otras 2 bebidas no se excretó un volumen equivalente al consumido sino hasta las 3 horas (Mayol-Soto y Aragón-Vargas, sin publicar).

Dicho estudio se realizó con el objeto de detectar un posible efecto diurético en el agua de jamaica y se utilizó agua pura como parámetro para determinar este efecto, siendo consideradas como diuréticos todas aquellas bebidas que se excretaran en mayor proporción que el agua pura. Así, se concluyó que el agua de jamaica no presentó un efecto diurético en un período de 3 horas después de haber sido consumida.

Un diurético es "una medicina que aumenta la cantidad de orina producida al ayudar a los riñones a la eliminación de agua y sal” (United States Pharmacopeia. Complete Drug Reference, 1993, p.1403). No obstante, resulta complicado dar una definición operacional del efecto diurético de una bebida, dado que, además de las sustancias que pueda contener, existe un efecto mecánico del líquido por sí solo, sobre todo cuando los sujetos se encuentran euhidratados. Así, si la persona está bien hidratada, cualquier bebida ocasionará un "efecto diurético" dado que el organismo no requiere el líquido en ese momento. Sin embargo, fue interesante observar que aún así, tanto la bebida deportiva como el agua de jamaica se conservaron durante más tiempo que el agua pura (Mayol-Soto y Aragón-Vargas, sin publicar).

Así, para efectos de este estudio, también se utilizó como parámetro al agua pura, pero se valoró el grado de conservación del líquido en una situación en la cual el cuerpo sí necesitó ese líquido, es decir, después de un estado de deshidratación inducido por el ejercicio.

Por lo tanto, el propósito del presente estudio fue determinar el grado de conservación del agua de jamaica, comparándola con una bebida deportiva y el agua pura, después de un estado de deshidratación inducido por el ejercicio, con el fin de determinar si el agua de jamaica es adecuada o no para la rehidratación después del ejercicio.

\section{METODOLOGÍA}

\section{Sujetos}

En este estudio participaron 16 varones, adultos jóvenes, entre los 17 y 23 años de edad, físicamente activos, es decir, que practicaban una actividad física por lo menos 3 veces a la semana en sesiones de 30 minutos como mínimo.

Los sujetos dieron su consentimiento por escrito después de haber conocido los riesgos y beneficios de su participación y el proyecto fue aprobado por el Comité Etico Científico de la Universidad de Costa Rica.

El tamaño de la muestra se determinó con base en estudios previos.

Figura 1. Protocolo experimental.

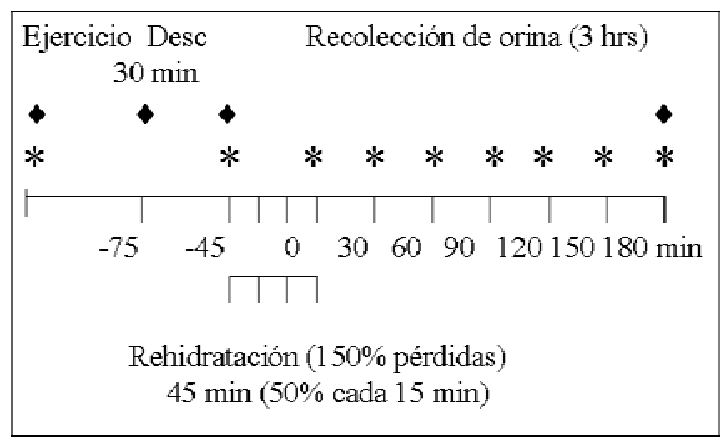

* Muestras de orina (determinación de volumen, densidad y color).

- Toma de peso (también se tomó el peso durante el ejercicio las veces que fuera necesario hasta alcanzar el $2 \%$ de deshidratación). 


\section{Instrumentos}

Se utilizó una báscula electrónica Acculab SV-100 con una precisión de 0.02 kg (20g), para medir el peso corporal.

Para la recolección de muestras de orina, se utilizaron recipientes de plástico con capacidad de 1 litro, y se midió el volumen de orina con probetas de diferentes capacidades en múltiplos de $0.5 \mathrm{ml}, 1 \mathrm{ml}, 5$ $\mathrm{ml} \mathrm{y} 10 \mathrm{ml}$.

Se utilizó un densímetro con una precisión de 0.001 para determinar la densidad de la orina y una escala de color para evaluar la concentración de la orina (Armstrong, 1998), la cual va de 1 a 7, siendo 1 la orina más clara y 7 la más oscura. También se utilizó una escala de "sensación de llenura” de 1 a 5, siendo 1 vacío y 5 extremadamente lleno. Además, para tener control sobre las condiciones iniciales de los sujetos en cada día, se realizó un "Recordatorio de 24 horas" en el cual se registraron todos los alimentos y bebidas que el sujeto consumió el día anterior a cada prueba, para determinar el consumo de agua y sodio. También se realizó un cuestionario de "Control de requisitos para la participación en el estudio", para determinar si los participantes siguieron todas las instrucciones que se les pidieron.

\section{Procedimientos}

Las pruebas experimentales se llevaron a cabo en el Laboratorio de Ambiente Controlado de la Escuela de Educación Física de la Universidad de Costa Rica, en tres días distintos, separados por una semana. Se realizaron las pruebas en el mismo día de la semana para mejorar la probabilidad de que los sujetos se presentaran en condiciones similares a los diferentes tratamientos.

El protocolo consistió en someter a los sujetos a una sesión de ejercicio intermitente dentro de un cuarto de clima controlado, con una temperatura de $30-35^{\circ} \mathrm{C}$ y una humedad relativa de $85 \%$, para inducir una pérdida de sudor equivalente al $2 \%$ de la masa corporal, seguido por la ingestión de una bebida en una cantidad equivalente al $150 \%$ de la pérdida. Al término del período de rehidratación, se tomaron muestras de orina durante las 3 horas siguientes.

Los participantes fueron sometidos a tres tratamientos con diferente tipo de bebida: agua pura, bebida deportiva y agua de jamaica. El orden de los tratamientos se decidió aleatoriamente y se procuró que los sujetos presentaran las mismas condiciones de hidratación en todas las pruebas. Para esto, se pidió a los sujetos que 24 horas antes de las pruebas evitaran el consumo de bebidas que tuvieran cafeína o alcohol, evitaran alimentos que tuvieran mucha sal y consumieran al menos 3 litros de líquido; así mismo, los sujetos debieron evitar el ejercicio vigoroso un día antes de las pruebas.

Los sujetos se presentaron los días de las pruebas a las 7 a.m. en ayunas y con una muestra de la primera orina del día, la cual fue utilizada para determinar el estado de hidratación del sujeto, por medio de la medición de la densidad y el color. Posteriormente, los sujetos completaron el cuestionario de "Control de requisitos para la participación en el estudio", consumieron un desayuno estandarizado y esperaron $45 \mathrm{~min}$ para iniciar el ejercicio. El desayuno estandarizado consistió en: $240 \mathrm{ml}$ de agua pura, 60 g de pan blanco (2 rebanadas) con 10 g de margarina y $240 \mathrm{ml}$ de jugo de naranja. Este desayuno aportó 345 kcal, 56.5 g de carbohidratos, $6.8 \mathrm{~g}$ de proteína, $10.6 \mathrm{~g}$ de grasa, $427 \mathrm{mg}$ de sodio y $531.7 \mathrm{ml}$ de agua (tomando en cuenta el agua de los líquidos y alimentos, así como el agua metabólica).

Después de vaciar la vejiga y tomar el peso corporal sin ropa, se inició la sesión de ejercicio, la cual consistió en un ejercicio intermitente en bicicleta a una intensidad entre el $65-70 \%$ de la frecuencia cardiaca máxima, dentro del cuarto de clima controlado. La frecuencia cardiaca máxima se calculó con la fórmula 219-(0.8xedad) 
(Aragón-Vargas, Schork y Eddington, 1993). El patrón de ejercicio consistió en 20 min de ejercicio y 10 min de descanso, en los cuales se tomó el peso corporal para determinar las pérdidas por deshidratación; este patrón se repitió hasta alcanzar un nivel de deshidratación equivalente al $2 \%$ del peso corporal.

Después de realizar el ejercicio, se dejó a los sujetos descansar durante $30 \mathrm{~min}$ fuera del cuarto a temperatura ambiente, con el fin de que se estabilizaran y disminuyera la tasa de sudoración. Posteriormente, los sujetos vaciaron la vejiga y se les tomó el peso para calcular las pérdidas totales $\mathrm{y}$ determinar la cantidad de líquido que debió ingerir cada sujeto (150\% de las pérdidas).

Durante los siguientes $45 \mathrm{~min}$, los sujetos consumieron la bebida que les correspondía, la cual fue dividida en 3 partes iguales, cada una de las cuales fue consumida en períodos de 15 minutos.

El agua de jamaica se preparó un día antes de ser utilizada y se mantuvo en refrigeración. Se utilizaron $10 \mathrm{~g}$ de flor de jamaica por litro de agua, los cuales se dejaron hervir durante 5 minutos, sacando inmediatamente las flores del agua. Se le agregó azúcar en una concentración de 6\% (60 g de azúcar por litro) para procurar que tuviera una concentración de azúcar similar a la de la bebida deportiva, y se dejó enfriar. Todas las bebidas estuvieron a la misma temperatura $\left(14-17^{\circ} \mathrm{C}\right)$.

Al terminar de consumir la bebida, los sujetos reportaron la "sensación de llenura", utilizando una escala que va de 1 a 5, siendo 1 vacío y 5 extremadamente lleno.

Se inició la recolección de orina al término del período de rehidratación (min 0) y posteriormente cada $30 \mathrm{~min}$ hasta completar las 3 horas. Se determinó el volumen, la densidad y el color de cada muestra. En la Figura 1 se muestra un resumen del protocolo experimental.

Se realizó la entrevista para el "Recordatorio de 24 hrs.", entre la toma de una muestra y otra y se obtuvo el peso del sujeto al final de la prueba.

\section{Análisis estadístico}

Para el análisis de los datos se utilizó el programa estadístico SPSS versión 8.0. Se obtuvo la estadística descriptiva para todas las variables en los tres tratamientos. La producción de orina se analizó como la proporción (\%) del volumen ingerido que fue excretada en cada medición. El balance neto de fluidos se determinó a partir de la diferencia de peso entre el inicio de la prueba (antes del ejercicio) y al final de las 3 horas de recolección de orina.

Se aplicó un ANOVA de 1 vía de medidas repetidas para analizar la densidad y el color de la orina pretratamiento, el consumo de agua y sodio en las 24 horas previas a cada prueba, el tiempo de ejercicio, el peso perdido durante el ejercicio (kg y \%), el volumen de bebida consumido, la temperatura de la bebida, la sensación de llenura y la producción total de orina.

Asimismo, se aplicó un ANOVA de 2 vías de medidas repetidas en ambos factores $(7 \times 3)$ siete mediciones por tres tratamientos, para analizar la producción parcial de orina, la producción acumulada de orina, la densidad y el color de la orina. En los análisis de densidad y color solo se incluyeron aquellos sujetos que dieron todas las muestras de orina.

El balance neto de fluidos se analizó con un ANOVA de 2 vías de medidas repetidas en ambos factores (2x3): dos mediciones (peso inicial y final) por tres bebidas.

\section{RESULTADOS}

El análisis químico de las bebidas que se utilizaron en este estudio se presenta en el Cuadro 1.

Condiciones iniciales. A partir de las respuestas del Instrumento \#2 se observó que todos los sujetos siguieron las indicaciones de alimentación e hidratación que se les dieron para los días anteriores a cada prueba. 
Cuadro 1. Composición del agua de jamaica, bebida deportiva y agua pura por cada litro de bebida.

\begin{tabular}{lccc}
\hline & $\begin{array}{c}\text { Agua de } \\
\text { jamaica }\end{array}$ & $\begin{array}{c}\text { Bebida } \\
\text { deportiva }\end{array}$ & $\begin{array}{c}\text { Agua } \\
\text { pura }\end{array}$ \\
\hline CHO totales (g) & 52 & 58.33 & - \\
Sodio (mg) & 4 & 458.33 & Trazas \\
Potasio (mg) & 150 & 125 & Trazas \\
PH & 3.66 & 3.3 & - \\
Cafeína & - & - & - \\
\hline
\end{tabular}

$\mathrm{Al}$ analizar la primera orina del día (Cuadro 2) no se encontraron diferencias estadísticamente significativas en la densidad $(p=0.540)$ ni el color $(p=0.767)$ de la orina, lo cual parece indicar que los sujetos presentaron las mismas condiciones de hidratación para los 3 tratamientos.

Por medio del análisis de la dieta del día anterior se mostró que no hubo diferencia estadísticamente significativa en el consumo de sodio previo a los tres tratamientos ( $p=$ 0.705). No obstante, no se logró que los sujetos tuvieran el mismo consumo de agua en las 24 horas previas a los tres tratamientos $\mathrm{y}$ tuvieron un consumo significativamente menor $(p=0.003)$ un día antes del tratamiento con bebida deportiva (Cuadro 2). Sin embargo, no se espera que esta diferencia altere los resultados, ya que en los tres casos los sujetos consumieron una cantidad mayor a los 3 litros de líquido, lo cual fue suficiente para garantizar un adecuado estado de hidratación, además de que hubo suficiente tiempo como para excretar cualquier exceso de líquido antes de reportarse al laboratorio, como puede observarse en el peso inicial.
No se encontraron diferencias estadísticamente significativas en el peso inicial de los sujetos ( $p=0.091)$ (Cuadro 2).

Normalización, deshidratación y rehidratación. El tiempo promedio de ejercicio fue de 60-61 min y no existieron diferencias estadísticamente significativas ( $p$ $=0.750$ ) entre los tres tratamientos.

No se encontraron diferencias estadísticamente significativas en el peso perdido durante el ejercicio ( $p=0.245)$, ni en el peso perdido total $(p=0.272)$ en el cual se tomó en cuenta las pérdidas por sudoración de los 30 min de descanso (Cuadro 3).

No hubo diferencias estadísticamente significativas en el volumen consumido ( $p=$ 0.285 ) y en la temperatura de la bebida ( $p=$ 0.920) entre los tres tratamientos (Cuadro 3 ).

En cuanto a la "sensación de llenura" (Cuadro 3), se observó que a pesar de haber consumido la misma cantidad de líquido en los tres tratamientos, los sujetos reportaron sentirse más llenos con el agua pura que con las otras 2 bebidas, aunque esta diferencia no alcanzó significancia estadística $(p=0.059)$.

Producción de orina. Después de 3 horas, la producción total de orina fue de $1429.41 \pm 414.15 \mathrm{ml}$ con el agua de jamaica, $1301.66 \pm 252.79$ con la bebida deportiva y $1483.44 \pm 330.8 \mathrm{ml}$ con el agua pura; no obstante, estas diferencias no fueron estadísticamente significativas $(p=0.084)$. En el Gráfico 1 se muestra la producción total de orina expresada como \% del volumen ingerido.

Cuadro 2. Condiciones pretratamiento (media \pm d.e.). AJ: agua de jamaica, BD: bebida deportiva, AP: agua pura. * Valor significativamente diferente de los otros dos $(p<0.05)$.

\begin{tabular}{lccccc}
\hline & $\begin{array}{c}\text { Densidad de } \\
\text { orina }\end{array}$ & $\begin{array}{c}\text { Color de } \\
\text { orina }\end{array}$ & $\begin{array}{c}\text { Consumo de } \\
\text { sodio } \\
\mathbf{2 4 ~} \mathbf{~}(\mathbf{m g})\end{array}$ & $\begin{array}{c}\text { Consumo de agua } \\
\mathbf{2 4} \mathbf{~ h}(\mathbf{m l})\end{array}$ & $\begin{array}{c}\text { Peso inicial } \\
(\mathbf{k g})\end{array}$ \\
\hline Agua de jamaica & $1.015 \pm 0.008$ & $2.88 \pm 0.96$ & $2376.13 \pm 1044.74$ & $4226.71 \pm 966.57$ & $69.6 \pm 10.69$ \\
Bebida deportiva & $1.013 \pm 0.008$ & $2.81 \pm 1.05$ & $2169.97 \pm 817.19$ & $3470.91 \pm 705.59 *$ & $69.3 \pm 10.77$ \\
Agua pura & $1.014 \pm 0.009$ & $2.69 \pm 0.95$ & $2408.81 \pm 622.02$ & $3998.45 \pm 759.28$ & $69.4 \pm 10.83$ \\
\hline
\end{tabular}


Cuadro 3. Pérdida de peso por deshidratación y rehidratación (media \pm d.e.).

\begin{tabular}{|c|c|c|c|c|c|c|c|}
\hline \multirow[b]{2}{*}{$\begin{array}{l}\text { Tipo de } \\
\text { bebida }\end{array}$} & \multicolumn{2}{|c|}{$\begin{array}{c}\text { Peso perdido por } \\
\text { ejercicio }\end{array}$} & \multicolumn{2}{|c|}{$\begin{array}{c}\text { Peso perdido } \\
\text { total }\end{array}$} & \multicolumn{3}{|c|}{ Rehidratación } \\
\hline & Peso (kg) & \% peso & Peso (kg) & $\%$ peso & $\begin{array}{c}\text { Volumen } \\
\text { consumido } \\
(\mathrm{ml})\end{array}$ & $\begin{array}{c}\text { Temp de } \\
\text { bebida } \\
\left({ }^{\circ} \mathrm{C}\right)\end{array}$ & $\begin{array}{c}\text { Sensación de } \\
\text { Ilenura }\end{array}$ \\
\hline $\begin{array}{l}\text { Agua de } \\
\text { jamaica }\end{array}$ & $1.40 \pm 0.25$ & $2.01 \pm 0.16$ & $1.62 \pm 0.28$ & $2.34 \pm 0.30$ & $2425.19 \pm 397.16$ & $15.03 \pm 0.96$ & $3.56 \pm 0.81$ \\
\hline $\begin{array}{l}\text { Bebida } \\
\text { deportiva }\end{array}$ & $1.38 \pm 0.22$ & $2.00 \pm 0.12$ & $1.58 \pm 0.24$ & $2.29 \pm 0.24$ & $2361.19 \pm 351.06$ & $15.13 \pm 1.01$ & $3.50 \pm 0.89$ \\
\hline $\begin{array}{l}\text { Agua } \\
\text { pura }\end{array}$ & $1.42 \pm 0.25$ & $2.05 \pm 0.15$ & $1.64 \pm 0.27$ & $2.37 \pm 0.24$ & $2444.69 \pm 398.03$ & $15.00 \pm 1.08$ & $4.13 \pm 0.62$ \\
\hline
\end{tabular}

En el Cuadro 4 se observan los valores promedio de volumen, densidad y color de cada una de las muestras de orina.

En el Gráfico 2 se muestra la producción parcial de orina en cada una de las mediciones. Se observaron diferencias estadísticamente significativas a los $60 \mathrm{~min}$, siendo la bebida deportiva la que se excretó en mayor cantidad $(15.17 \pm 3.32 \%)$ que las otras dos bebidas $(12.90 \pm 2.71 \%$ y $12.94 \pm$ $4.26 \%$, del agua de jamaica y el agua pura, respectivamente). No obstante, aunque en un inicio se excretó en mayor proporción la bebida deportiva, la producción de orina con esta bebida fue significativamente menor que las otras dos bebidas a los $150 \min (5.11 \pm$ $3.35 \%$, vs. $8.58 \pm 3.61 \%$ del agua de jamaica y $8.18 \pm 4.56 \%$ del agua pura) y también fue significativamente menor que el agua de jamaica a los $180 \min (3.63 \pm 2.99 \%$ de la bebida deportiva y $6.65 \pm 3.38 \%$ del agua de jamaica). En el Cuadro 4 se pueden observar los valores del volumen de orina excretados en cada medición.

En el Gráfico 3 se observa la producción acumulada de orina, la cual fue significativamente mayor a los 60 min con la bebida deportiva (24.23 $\pm 6.54 \%)$ que con las otras 2 bebidas ( $20.01 \pm 7.44 \%$ del agua de jamaica y $20.77 \pm 6.79 \%$ del agua pura), mientras que a los 90 min esta diferencia solo se dio entre la bebida deportiva (38.05 \pm 9.26\%) y el agua de jamaica (32.29 \pm $10.87 \%)$.

Se encontraron diferencias estadísticamente significativas en la densidad $(p=0.006)$ y el color $(p=0.002)$ de la orina entre las 3 bebidas. En el Cuadro 4 se muestran los valores promedio de densidad y color de cada una de las muestras. En los Gráficos 4 y 5 se puede observar que la densidad y el color siguen el mismo comportamiento, es decir, que a mayor densidad, la orina presentará un color más oscuro, mientras que a menor densidad la orina será más clara. Además, se observa una relación inversa entre estas dos variables y el volumen (Gráfico 2), pues a mayor volumen, menor densidad y color, y viceversa.

Gráfico 1. Producción de orina después de 3 horas de tratamiento, expresada como \% del volumen ingerido (media).

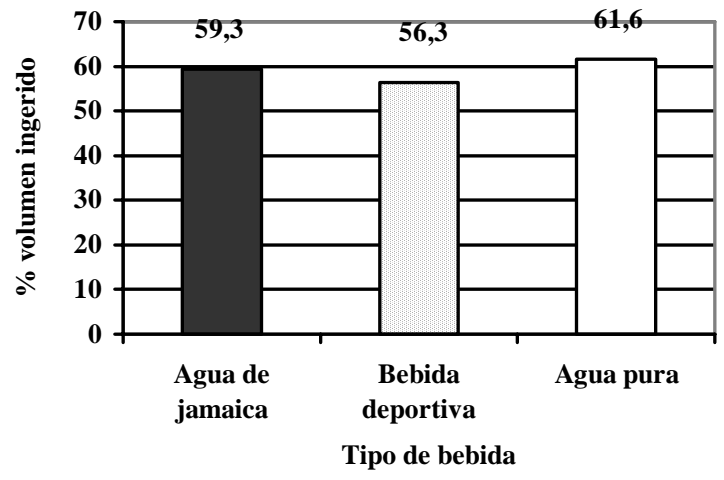

A los $0 \mathrm{~min}$, la orina producida por la bebida deportiva fue significativamente más densa que la producida por el agua pura; y a los 180 min también se observó una mayor densidad de la orina producida por esta bebida que la de las otras dos bebidas (Cuadro 4 y Gráfico 4). 
Gráfico 2. Producción parcial de orina (media). Bebida $p=0.084$, Tiempo $p<0.001$, Interacción entre bebida y tiempo $p<0.001$. *Diferencia entre bebida deportiva y agua pura. $\downarrow$ Diferencia entre bebida deportiva y agua de jamaica.

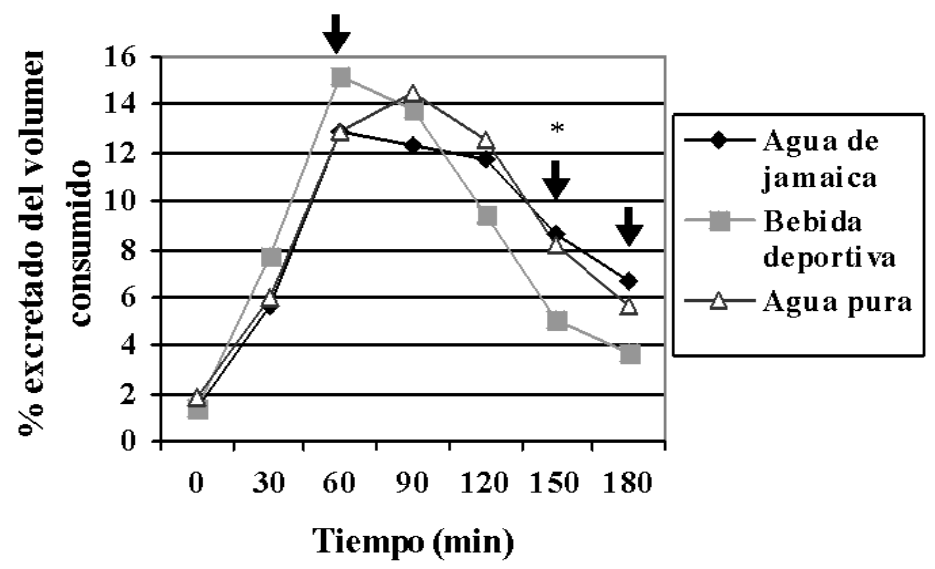

Gráfico 3. Producción acumulada de orina en cada medición (media). Bebida $p=0.322$, Tiempo $p<0.001$, Interacción entre bebida y tiempo $p<0.001$. *Diferencia entre bebida deportiva y agua pura. $\downarrow$ Diferencia entre bebida deportiva y agua de jamaica.

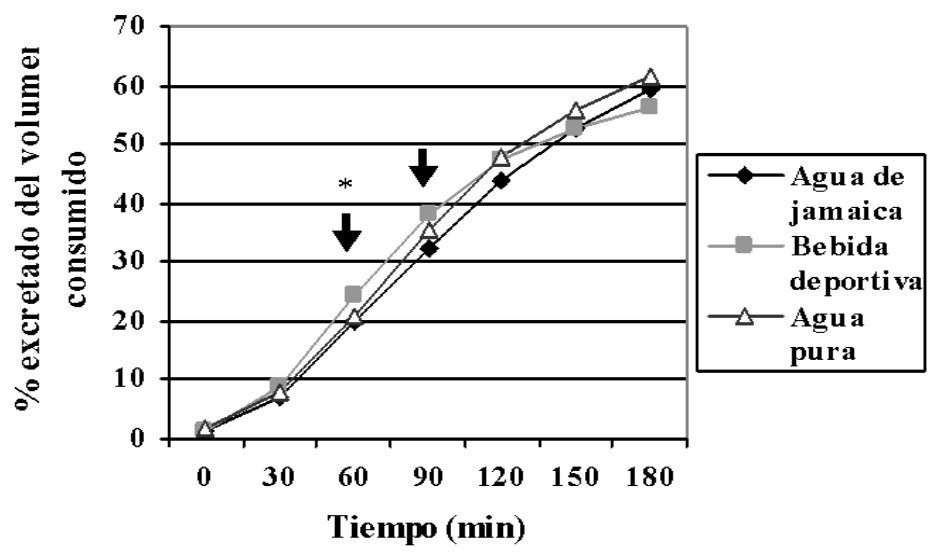

En cuanto al color, se observó que la orina producida por la bebida deportiva fue significativamente más oscura a los 150 min que la producida por el agua de jamaica y a los $180 \mathrm{~min}$ fue significativamente más oscura que la de las otras dos bebidas (Cuadro 4 y Gráfico 5).

En el Gráfico 6 se puede observar que los sujetos perdieron y recuperaron la misma cantidad de peso en los tres tratamientos; que la pérdida de peso a través del tiempo fue muy similar y que los sujetos entraron en balance negativo de fluido (tomando en cuenta solo las pérdidas por orina) después de los 90 min en los tres tratamientos. Por medio del análisis del balance neto de fluidos se observó que aunque hubo diferencia estadísticamente significativa entre el peso inicial y final ( $p$ $<0.0005$ ), no se encontraron diferencias significativas entre bebidas $(p=0.178)$, ni hubo interacción entre la bebida y el tiempo $(p=0.150)$. Al final del estudio los sujetos terminaron con un déficit de peso de $890 \mathrm{~g}$, 730 g y 840 g para el agua de jamaica, bebida deportiva y agua pura, 
respectivamente. En el Cuadro 5 se muestra la procedencia de las pérdidas de líquido. Así, el balance neto de fluidos fue igual para los tres tratamientos y en los tres casos el peso final fue significativamente menor que el peso inicial.

Cuadro 4. Volumen, densidad y color de cada una de las muestras de orina. Los valores representan la media ( \pm d.e.).

\begin{tabular}{|c|c|c|c|c|c|c|c|c|}
\hline \multirow[b]{2}{*}{ Variable } & \multirow[b]{2}{*}{ Bebida } & \multicolumn{7}{|c|}{ Tiempo de recolección de orina (min) } \\
\hline & & $\mathbf{0}$ & 30 & 60 & 90 & 120 & 150 & 180 \\
\hline \multirow{3}{*}{$\begin{array}{c}\text { Volumen } \\
\text { parcial } \\
(\mathbf{m l}) \\
(\mathbf{n}=16)\end{array}$} & $\begin{array}{l}\text { Agua de } \\
\text { jamaica }\end{array}$ & $\begin{array}{c}34.47 \\
( \pm 35.12)\end{array}$ & $\begin{array}{c}140.44 \\
( \pm 123.91)\end{array}$ & $\begin{array}{c}310.13 \\
( \pm 74.15)\end{array}$ & $\begin{array}{c}293.47 \\
( \pm 108.54)\end{array}$ & $\begin{array}{c}281.81 \\
( \pm 96.1)\end{array}$ & $\begin{array}{c}208.53 \\
( \pm 91.17) \\
\end{array}$ & $\begin{array}{c}160.56 \\
( \pm 83.56)\end{array}$ \\
\hline & $\begin{array}{l}\text { Bebida } \\
\text { deportiva }\end{array}$ & $\begin{array}{c}31.00 \\
( \pm 16.02)\end{array}$ & $\begin{array}{c}178.50 \\
( \pm 111.59)\end{array}$ & $\begin{array}{c}351.59 \\
( \pm 65.99)^{*}\end{array}$ & $\begin{array}{c}316.69 \\
( \pm 101.61)\end{array}$ & $\begin{array}{c}220.91 \\
( \pm 97.15)\end{array}$ & $\begin{array}{c}120.31 \\
( \pm 80.35) *\end{array}$ & $\begin{array}{c}82.66 \\
( \pm 68.14)\end{array}$ \\
\hline & Agua pura & $\begin{array}{c}45.47 \\
( \pm 42.85)\end{array}$ & $\begin{array}{c}145.63 \\
( \pm 84.78)\end{array}$ & $\begin{array}{c}310.03 \\
( \pm 98.85)\end{array}$ & $\begin{array}{c}349.94 \\
( \pm 91.44)\end{array}$ & $\begin{array}{c}300.63 \\
( \pm 92.05)\end{array}$ & $\begin{array}{c}195.72 \\
( \pm 103.33)\end{array}$ & $\begin{array}{c}136.03 \\
( \pm 70.39)\end{array}$ \\
\hline \multirow{3}{*}{$\begin{array}{c}\text { Densidad } \\
(n=13)\end{array}$} & $\begin{array}{l}\text { Agua de } \\
\text { jamaica }\end{array}$ & $\begin{array}{c}1.020 \\
( \pm 0.007)\end{array}$ & $\begin{array}{c}1.002 \\
( \pm 0.003)\end{array}$ & $\begin{array}{c}1.000 \\
( \pm 0.000)\end{array}$ & $\begin{array}{c}1.000 \\
( \pm 0.000)\end{array}$ & $\begin{array}{c}1.000 \\
( \pm 0.000)\end{array}$ & $\begin{array}{c}1.000 \\
( \pm 0.000) \\
\end{array}$ & $\begin{array}{c}1.001 \\
( \pm 0.003)\end{array}$ \\
\hline & $\begin{array}{l}\text { Bebida } \\
\text { deportiva }\end{array}$ & $\begin{array}{c}1.023 \\
( \pm 0.006) *\end{array}$ & $\begin{array}{c}1.002 \\
( \pm 0.002)\end{array}$ & $\begin{array}{c}1.000 \\
( \pm 0.000)\end{array}$ & $\begin{array}{c}1.000 \\
( \pm 0.001)\end{array}$ & $\begin{array}{c}1.000 \\
( \pm 0.001)\end{array}$ & $\begin{array}{c}1.002 \\
( \pm 0.003)\end{array}$ & $\begin{array}{c}1.006 \\
( \pm 0.004) *\end{array}$ \\
\hline & Agua pura & $\begin{array}{c}1.018 \\
( \pm 0.005) \\
\end{array}$ & $\begin{array}{c}1.003 \\
( \pm 0.006) \\
\end{array}$ & $\begin{array}{c}1.001 \\
( \pm 0.003) \\
\end{array}$ & $\begin{array}{c}1.000 \\
( \pm 0.000)\end{array}$ & $\begin{array}{c}1.000 \\
( \pm 0.000) \\
\end{array}$ & $\begin{array}{c}1.001 \\
( \pm 0.002) \\
\end{array}$ & $\begin{array}{c}1.001 \\
( \pm 0.003) \\
\end{array}$ \\
\hline \multirow{3}{*}{$\begin{array}{c}\text { Color } \\
(n=13)\end{array}$} & $\begin{array}{l}\text { Agua de } \\
\text { jamaica }\end{array}$ & $\begin{array}{c}3.31 \\
( \pm 0.75) \\
\end{array}$ & $\begin{array}{c}1.54 \\
( \pm 0.66) \\
\end{array}$ & $\begin{array}{c}1.00 \\
( \pm 0.00)\end{array}$ & $\begin{array}{c}1.00 \\
( \pm 0.00)\end{array}$ & $\begin{array}{c}1.00 \\
( \pm 0.00)\end{array}$ & $\begin{array}{c}1.00 \\
( \pm 0.00)\end{array}$ & $\begin{array}{c}1.08 \\
( \pm 0.28) \\
\end{array}$ \\
\hline & $\begin{array}{l}\text { Bebida } \\
\text { deportiva }\end{array}$ & $\begin{array}{c}4.08 \\
( \pm 1.12) \\
\end{array}$ & $\begin{array}{c}1.54 \\
( \pm 0.52) \\
\end{array}$ & $\begin{array}{c}1.00 \\
( \pm 0.00) \\
\end{array}$ & $\begin{array}{c}1.08 \\
( \pm 0.28) \\
\end{array}$ & $\begin{array}{c}1.15 \\
( \pm 0.38) \\
\end{array}$ & $\begin{array}{c}1.62 \\
( \pm 0.65)\end{array}$ & $\begin{array}{c}2.15 \\
( \pm 0.90) *\end{array}$ \\
\hline & Agua pura & $\begin{array}{c}3.38 \\
( \pm 0.96)\end{array}$ & $\begin{array}{c}1.38 \\
( \pm 0.65)\end{array}$ & $\begin{array}{c}1.08 \\
( \pm 0.28)\end{array}$ & $\begin{array}{c}1.00 \\
( \pm 0.00)\end{array}$ & $\begin{array}{c}1.08 \\
( \pm 0.28)\end{array}$ & $\begin{array}{c}1.23 \\
( \pm 0.44)\end{array}$ & $\begin{array}{c}1.38 \\
( \pm 0.65)\end{array}$ \\
\hline
\end{tabular}

* Diferencia estadísticamente significativa con el agua pura $(p<0.05)$.

- Diferencia estadísticamente significativa con el agua de jamaica $(p<0.05)$.

\section{DISCUSION}

El objetivo del presente estudio fue determinar si el agua de jamaica es una bebida adecuada para la rehidratación después del ejercicio. Al parecer el agua de jamaica no presenta un efecto diurético, pues no hubo diferencia estadísticamente significativa en el volumen de orina producido después de 3 horas $(p=0.084)$ al compararla con las otras dos bebidas. Más bien, la tendencia fue a excretarse en menor proporción que el agua pura (Gráfico 1).

\section{Cuadro 5. Pérdidas de peso por orina y sudoración después de 3 horas de tratamiento ( $n=16)$ (media \pm d.e.).}

\begin{tabular}{lcccc}
\hline \multicolumn{1}{c}{ Tipo de bebida } & Peso final $(\mathbf{k g})$ & $\begin{array}{c}\text { Déficit de peso } \\
(\mathbf{k g})\end{array}$ & $\begin{array}{c}\text { Peso perdido por } \\
\text { orina }(\mathbf{k g})\end{array}$ & $\begin{array}{c}\text { Peso perdido por } \\
\text { sudor }(\mathbf{k g})\end{array}$ \\
\hline Agua de jamaica & $68.71 \pm 10.62$ & $-0.89 \pm 0.41$ & $1.43 \pm 0.41$ & $0.26 \pm 0.30$ \\
Bebida deportiva & $68.56 \pm 10.87$ & $-0.73 \pm 0.24$ & $1.30 \pm 0.25$ & $0.21 \pm 0.12$ \\
Agua pura & $68.58 \pm 10.86$ & $-0.84 \pm 0.31$ & $1.48 \pm 0.33$ & $0.17 \pm 0.08$ \\
\hline
\end{tabular}

$\mathrm{Al}$ analizar la producción de orina en cada medición (Gráfico 2) se puede observar que la producción parcial de orina fue significativamente mayor con la bebida deportiva en un inicio (a los $60 \mathrm{~min}$ ), pero posteriormente disminuyó el volumen de orina producido, siendo a partir de los 120 min la bebida que se excretó en menor 
proporción. En el Gráfico 3 se puede observar la tendencia de cada una de las 3 bebidas al final del estudio, y se aprecia cómo la bebida deportiva ya casi no se excreta a partir de los $150 \mathrm{~min}$, mientras que el agua pura y el agua de jamaica presentan una tendencia a seguirse excretando. Así, la bebida deportiva se empieza a conservar más que las otras dos bebidas después de 2 horas y media de haber sido consumida.

Al parecer el agua de jamaica no presenta un efecto diurético después de un estado de deshidratación inducido por el ejercicio y pareciera ser que el proceso de rehidratación es igual con cualquiera de las tres bebidas, después de 3 horas de haber sido consumidas.

Sin embargo, aunque no se encontraron diferencias estadísticamente significativas en la producción parcial $(p=$ $0.084)$ y acumulada ( $p=0.322)$ de la orina producida por las tres bebidas, se encontraron diferencias significativas en la densidad $(p=0.006)$ y el color $(p=0.002)$ de la orina, además de que se encontró una interacción entre cada una de estas cuatro variables (producción parcial de orina, producción acumulada de orina, densidad y color) y el tiempo ( $p<0.001)$; así, la cantidad, la densidad y el color de la orina excretada en cada instante del tiempo va a depender del tipo de bebida. Por lo tanto, la dinámica de producción y la composición de la orina no es la misma para las tres bebidas, aunque al final del estudio la rehidratación haya sido casi igual con las tres bebidas.

El color, la osmolalidad y la gravedad específica de la orina son índices válidos del estado de hidratación, además de que se ha observado que un alto grado de deshidratación, el ejercicio y la rehidratación, no afectan la validez y sensibilidad de estos índices (Armstrong et al., 1998).

La osmolalidad es el número de partículas de soluto en $1 \mathrm{~kg}$ de solvente; mientras que la gravedad específica se define como el peso de un volumen de solución dividido por el peso de un volumen equivalente de agua destilada (Koeppen y Stanton, 2001). La densidad es el peso de un volumen de solución, por lo que es equivalente a la gravedad específica.

Gráfico 4. Densidad de orina en cada medición (media). Bebida $p=0.006$, Tiempo $p<0.001$, Interacción entre bebida y tiempo $p<0.001$. *Diferencia entre bebida deportiva y agua pura. $\downarrow$ Diferencia entre bebida deportiva y agua de jamaica. El primer punto corresponde a la muestra pretratamiento, la cual se analizó por separado, pero se incluye como punto de referencia.

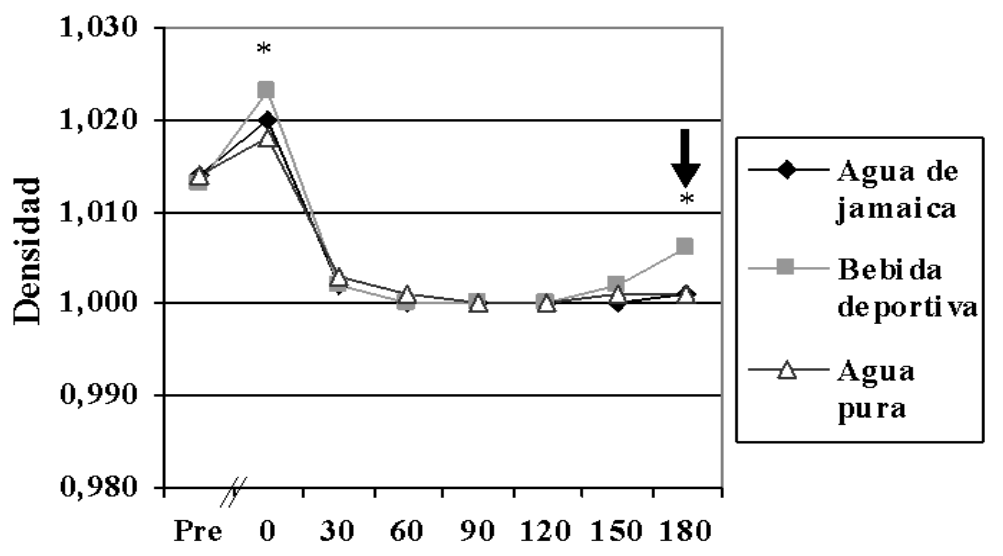

Tiempo (min) 
Gráfico 5. Color de la orina en cada medición $(\mathbf{n}=\mathbf{1 3})$. Bebida $p=0.002$, Tiempo $p<0.001$, Interacción entre bebida y tiempo $p<0.001$. * Diferencia entre bebida deportiva y el agua pura. $\downarrow$ Diferencia entre bebida deportiva y agua de jamaica. El primer punto corresponde a la muestra pretratamiento, la cual se analizó por separado, pero se incluye como punto de referencia.

$\begin{array}{llllllll}\text { Pre } & 0 & 30 & 60 & 90 & 120 & 150 & 180\end{array}$

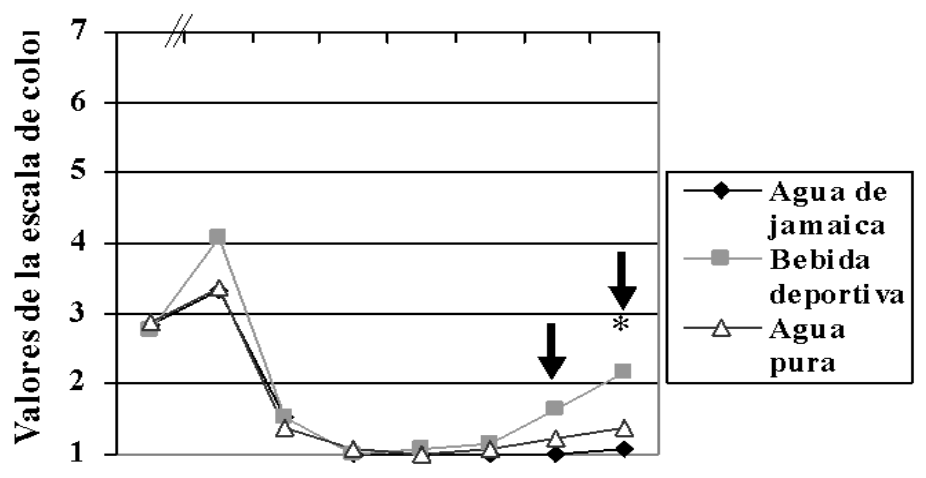

Tiempo (min)

Armstrong y colaboradores (1994) encontraron que el color de la orina presenta una alta correlación con la gravedad específica $(r=0.80, \quad p<0.0001)$ y la osmolalidad de la orina $(r=0.82, p<$ 0.0001). Aunque la osmolalidad de la orina depende del número de partículas en solución y la gravedad específica depende del número y masa de los solutos, estos índices pueden ser intercambiables $(r=0.97$, $p<0.001$ ); no obstante, es importante considerar que la varianza en esta correlación aumenta cuando la gravedad específica es mayor a 1.024 y la osmolalidad es mayor a $900 \mathrm{mOsm} \cdot \mathrm{kg}$ (Armstrong et al., 1994).

En este estudio se puede observar que los resultados de densidad y color tuvieron el mismo comportamiento, lo cual concuerda con los resultados de Armstrong y colaboradores (1994, 1998).

El color de la orina puede verse alterado por enfermedades, el consumo de suplementos vitamínicos y medicamentos. Además, se pueden presentar colores exóticos de orina (por ejemplo, rosa, rojo, verde, azul, negro) como resultado de pigmentos fisiológicos en concentraciones anormales, aditivos de los alimentos y crecimiento de bacterias en muestras almacenadas. En el presente estudio se controló que los sujetos no consumieran suplementos vitamínicos ni medicamentos durante el estudio, además de que ninguno presentó enfermedades, ni se observaron colores exóticos de orina; por otra parte, las muestras fueron analizadas en los $30 \mathrm{~min}$ siguientes a su recolección, por lo que se puede inferir que los resultados obtenidos a partir de este indicador son válidos.

Armstrong y colaboradores (1998) mencionan que las diferencias en la composición de los líquidos que se utilizan para la rehidratación, puede alterar tanto el volumen como la osmolalidad de la orina, y ponen como ejemplo los estudios de González-Alonso et al. (1992), y Maughan et al. (1994), donde se reporta que las bebidas que contienen cloruro de sodio o cloruro de potasio disminuyen el volumen y aumentan la osmolaridad de la orina, en comparación con el agua pura. Estos resultados concuerdan con los observados en el presente estudio, pues aunque se midieron otros indicadores (densidad y color, en vez de osmolalidad), los resultados son equivalentes, dada la alta correlación que se ha observado entre estos diferentes indicadores. En este estudio también se 
observó como la bebida deportiva (que contiene sodio) produjo a los 0, 150 y 180 min orinas más densas y más oscuras que las producidas por las otras 2 bebidas (Gráficos 4 y 5). Así, el agua de jamaica, a pesar de tener cierta cantidad de electrolitos, tuvo un comportamiento similar al agua pura. Esto se puede atribuir a su bajo contenido de sodio, en comparación con la bebida deportiva (4 mg/litro vs. $458 \mathrm{mg} / \mathrm{litro}$ ) ya que el contenido de potasio es muy similar en ambas bebidas (150 mg/litro y $125 \mathrm{mg} /$ litro, en el agua de jamaica y la bebida deportiva, respectivamente). En lo que se refiere a los carbohidratos totales, el agua de jamaica se manipuló para tener un contenido de carbohidratos (52 g/litro) muy similar al que naturalmente tiene la bebida deportiva (58 g/litro); como las cantidades eran prácticamente iguales, las diferencias tienen que obedecer al contenido de sodio.

Varios estudios demuestran que la concentración de sodio que aporta una bebida es el principal factor que determina el grado de conservación de líquido de la misma (Maughan et al., 1994; Maughan y Leiper, 1995; Shirreffs et al. 1996; Maughan, Leiper y Shirreffs, 1996; Maughan, 1998). Así, se ha demostrado que cuando solo se consume líquido en el proceso de rehidratación, la bebida debe aportar suficiente cantidad de sodio (50 mmol/litro) para que pueda ser conservada durante más tiempo (Maughan, 1998), aunque el sodio también puede obtenerse a partir de los alimentos (Maughan et al., 1996).

Así, es probable que la diferencia final en el grado de conservación entre la bebida deportiva y las otras dos bebidas no hayan sido significativas debido a que su contenido de sodio (20 mmol/litro) no es suficiente como para garantizar un mayor grado de conservación de líquido que las otras dos bebidas.

Gráfico 6. Cambios en el peso a través del tiempo. $\mathrm{I}=$ inicio, $\mathrm{D}=$ deshidratación, $\mathrm{R}=$ rehidratación. I y D son pesos tomados en báscula; R se determinó sumando el volumen ingerido al peso D; y los valores de 0 a 180 min son $\mathrm{R}$ menos las pérdidas acumuladas por orina y sudor. Se asumió que las pérdidas por sudoración fueron constantes entre la toma del peso después de la deshidratación y la toma del peso final.

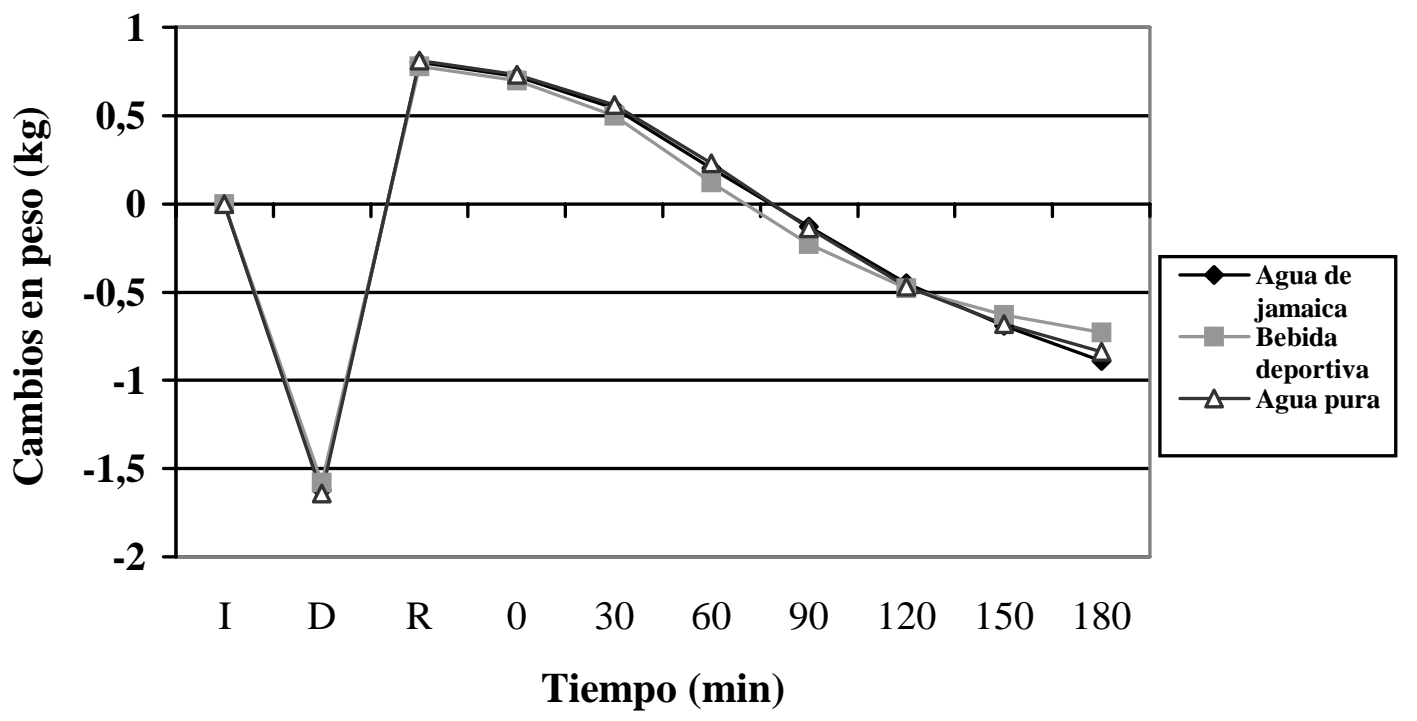


La densidad y el color de la orina son indicadores que nos pueden decir algo sobre un estado o un proceso. Así, en el caso del análisis de la primera orina del día, estos indicadores sirvieron para determinar el estado de hidratación de los sujetos. Sin embargo, las mediciones obtenidas después del período de rehidratación, nos indicaron cómo se fue dando el proceso de rehidratación. Es importante resaltar esta diferencia, pues podría haber confusión al interpretar los resultados. Por ejemplo, resulta contradictorio que a los $180 \mathrm{~min} \mathrm{la}$ orina producida por la bebida deportiva haya sido significativamente más densa y más oscura que la producida por las otras 2 bebidas, pues si esto se interpretara como un estado, se podría decir que los sujetos estaban más deshidratados con la bebida deportiva, siendo en realidad la que se excretó en menor cantidad. Así, si esta última medición se interpreta como parte de un proceso, la mayor densidad y el color más oscuro indicaron que el líquido se estaba conservando más dentro del organismo, favoreciendo el balance neto de líquidos en el cuerpo.

Esta tendencia de la bebida deportiva a ser excretada en menor proporción hacia el final del estudio, también se puede observar en el Gráfico 3, donde la pendiente de las líneas a los $180 \mathrm{~min}$ sugieren que si se hubiera continuado la recolección de orina durante más tiempo, es probable que se hubieran notado las diferencias en la producción total de orina entre la bebida deportiva que ya casi no se excreta y las otras 2 bebidas. También se observa que la pendiente del agua de jamaica está más inclinada que la del agua pura, lo cual, combinado con los resultados de color de la orina, que demuestran que la orina producida por el agua de jamaica estaba más diluida (más clara) que la producida por el agua pura, podrían indicar el "posible efecto diurético" del agua de jamaica; es decir, que a partir de ese momento se empezaran a producir orinas con un mayor volumen y una menor densidad y color con el agua de jamaica, ocasionando una mayor producción total de orina que con el agua pura. No obstante, se requeriría aumentar el tiempo de recolección de muestras de orina para poder confirmar esta hipótesis, y ver si las diferencias con el agua pura llegan a ser estadísticamente significativas. Sin embargo, la experiencia previa en estudios con alcohol (Shirreffs y Maughan, 1997) muestra que el efecto diurético del alcohol aparece a partir de los $120 \mathrm{~min}$; si el mecanismo fuera el mismo, el agua de jamaica debió mostrar un efecto diurético por lo menos a los 150 y 180 min.

En el presente estudio no se encontró un efecto diurético del agua de flor de jamaica (Hibiscus Sabdarifa L.) después de un estado de deshidratación inducido por el ejercicio, lo cuál concuerda con los resultados obtenidos anteriormente, donde se observó que el agua de jamaica tampoco ocasiona un efecto diurético en sujetos en estado de euhidratación después de 3 horas de haber sido consumida (Mayol, AragónVargas, sin publicar). Sin embargo, en varios textos se reitera que esta planta sí presenta este efecto (Atlas de las plantas de la Biblioteca Tradicional Mexicana, 1994; Towards a Caribbean Pharmacopoea, 1989; Fitoterapia, Vademécum de Prescripción: Plantas Medicinales, 1998) e incluso, en los empaques de flor de jamaica deshidratada se puede leer “..., las bebidas refrescantes (sin azúcar) son un excelente diurético y se recomienda a los enfermos febricitantes, a los débiles y a los biliosos”. No obstante, no existen estudios científicos que demuestren este efecto, y es probable que el efecto diurético se confunda con el efecto del volumen de líquido en sí. Sin embargo, en caso de existir alguna sustancia diurética no se sabe si este compuesto se puede desnaturalizar con la cocción, inhibiéndose así su efecto, o si el efecto pudiera presentarse después de 3 horas de haber sido consumida.

Un aspecto interesante que se presentó en el estudio, fue con respecto a la "sensación de llenura”, es decir, a la 
percepción que tuvieron los sujetos con respecto a la cantidad de líquido ingerido. Aunque estas diferencias no fueron estadísticamente significativas $(p=0.059)$, se observó que la mayoría de los sujetos se sintieron "más llenos" y les costó más trabajo consumir el agua pura que las otras dos bebidas, a pesar de haber consumido la misma cantidad de las tres bebidas. Esto no tuvo ninguna implicación en el presente estudio, dado que la rehidratación fue forzada, es decir, que los sujetos tuvieron que ingerir todo el líquido que se les dio, pero es probable que si el líquido se hubiera dado ad libitum (libre demanda) hubieran ingerido menor cantidad de agua pura que de las otras dos bebidas, lo cual afectaría el proceso de rehidratación.

Por lo tanto, a partir de los resultados obtenidos en el presente estudio, se puede concluir que el agua de jamaica no presentó un efecto diurético después de 3 horas de haber sido consumida por sujetos deshidratados por ejercicio. Aunque pareciera ser que la rehidratación es casi igual con las tres bebidas, la dinámica de producción y composición de la orina es diferente con cada una de las bebidas, además de que hacia el final del estudio se observó una tendencia de la bebida deportiva a conservarse más dentro del organismo.

\section{REFERENCIAS}

Aragón-Vargas, L.F., y Madriz-Dávila, K. (2000). Incomplete warm-climate post-exercise rehydration with water, coconut water or a sports drink. Med Sci Sports Exerc, 32(5), S-238.

Aragón-Vargas, L.F., Schork, M.A., y Eddington, D. (1993). Evaluation of conventional and new maximum heart rate prediction models for individuals. Med Sci Sports Exerc, 25(5), S-10.

Armstrong, L.E., Maresh, C.M., Castellani, J.W., Bergeron, M.F., Kenefick, R.W., LaGasse, K.E., y Riebe, D. (1994). Urinary indices of hydration status. Int J Sports Nut, 4: 265-279 (citado por Armstrong, et al, 1998).

Armstrong, L.E., Herrera, J.A., Hacker, F.T., Casa, D.J., Kavouras, S.A., y Maresh, C.M. (1998). Urinary indices during dehydration, exercise and rehydration. Int J Sports Nut, 8, 345-355.
Biblioteca de la Medicina Tradicional Mexicana (1994). Atlas de las Plantas de la Medicina Tradicional Mexicana. Tomo II. Instituto Nacional Indigenista. $1^{a}$. ed. México, D.F. pp: 849-850.

Colegio Oficial de Farmacéuticos de Bizkaina, Asociación Española de Médicos Naturistas (1998). Fitoterapia, Vademécum de Prescripción: Plantas Medicinales. Barcelona, España. Versión CD-room Wfitos 1.0.

González-Alonso, J., Heaps, C.L., Coyle, E.F. (1992). Rehydration after exercise with common beverages and water. Int $J$ sports Med, 13, 399-406.

Horswill, C.A. (1998). Effective fluid replacement. Int J Sports Nut, 8, 175-195.

Koeppen, B.M., y Stanton, B.A. (2001). Capítulo 1: Physiology of Body Fluids. En: Renal Physiology. $3^{\text {a }}$.ed. U.S.A.: Mosby, pp. 49-73.

Maughan, R.J., Owen, J.H., Shirreffs, S.M., y Leiper, J.B. (1994). Post-exercise rehydration in man: effects of electrolyte adition to ingested fluids. Eur J Appl Physiol, 69(3), 209-215.

Maughan, R.J., y Leiper, J.B. (1995). Sodium intake and post-exercise rehydration in man. Eur J Appl Physiol, 71(4), 311-319.

Maughan, R.J., Leiper, J.B., y Shirreffs, S.M. (1996). Rehydration and recovery after exercise. Sports Science Exchange, 9(3).

Maughan, R.J., Leiper, J.B., y Shirreffs, S.M. (1996). Restoration of fluid balance after exercise-induced dehydration: effects of food and fluid intake. Eur $J$ Appl Physiol, 73, 317-325.

Maughan, R.J. (1998). Restoration of water and electrolyte balance after exercise. Int J Sports Med, 19, S136-S138.

Mayol, L., y Aragón-Vargas, L.F. (2000). Comparación del grado de conservación de líquido producido por tres bebidas distintas: agua pura, bebida deportiva y agua de jamaica, bajo condiciones de reposo. Universidad de Costa Rica. Escuela de Educación Física y Deportes. Sin publicar.

Nadel, E.R., Mack, G.W., y Nose, H. (1990). Influence of fluid replacement beverages on body fluid homeostasis during exercise and recovery. En: Gisolfi, C.V., y Lamb, D.R., editors. Perspectives in Exercise Science and Sports Medicine. Vol. 3: Fluid Homeostasis During Exercise. Carmel, C.A.: Benchmark, pp: 181-205.

National Autonomous University of Honduras (1989). Towards a Caribbean Pharmacopoea. L. Robineau (editor). Honduras. pp. 23.

Shirreffs, S.M., Taylor, A.J., Leiper, J.B., y Maughan, R.J. (1996). Post-exercise rehydration in man: effects of volume consumed and sodium content of ingested fluids. Med Sci Sports Exerc, 28(10), 1260-1271.

Wilmore, J., y Costill, D. (1994). Physiology of Sports and Exercise. United States of America. Human Kinetics. 